\title{
Increased intima-media thickness of the carotid artery wall, normal blood pressure profile and normal left ventricular mass in subjects with primary hyperparathyroidism
}

V Nuzzo, L Tauchmanovà, F Fonderico, R Trotta ${ }^{1}$, M R Fittipaldi, D Fontana ${ }^{1}$, R Rossi, G Lombardi, B Trimarco ${ }^{1}$ and G Lupoli

Department of Molecular and Clinical Endocrinology and Oncology and ${ }^{1}$ Institute of Internal Medicine and Cardiology, University 'Federico II', Naples, Italy

(Correspondence should be addressed to G Lupoli, Dipartimento di Endocrinologia e Oncologia Molecolare e Clinica, Università degli Studi 'Federico II' di Napoli, Via S. Pansini 5, 80131 Napoli, Italy; Email: lupoli@unina.it)

\begin{abstract}
Objective: Despite the increasing evidence that primary hyperparathyroidism (PHPT) contributes to greater risk of cardiovascular morbidity and mortality, its exact role in the development of cardiovascular changes and its clinical significance are still controversial. Given the multiple influence of PHPT on the cardiovascular system, this study aimed to assess the effects of PHPT on blood pressure profile, and on features of the heart and arterial vessels in normotensive symptomless patients. Design: Twenty patients ( 8 males and 12 females) with a median age of 51.5 years (range 44 to 65 years) were evaluated and the results were compared with those of 20 controls matched for age, gender and body mass index. Patients' parathyroid hormone levels ranged from 172 to $454 \mathrm{pg} / \mathrm{ml}$ and Ca levels ranged from 11.4 to $13.5 \mathrm{mg} / \mathrm{dl}$. Fasting levels of glucose, insulin, total and high density lipoprotein cholesterol and triglycerides were within the normal range in all subjects recruited.

Methods: Twenty-four-hour blood pressure profile, left ventricle (LV) dimension and carotid artery anatomy were investigated, the latter two by ultrasonography.

Results: No difference was found between the patients and controls in blood pressure profile, when the following parameters were considered: supine systolic/diastolic pressure, average 24-h systolic, diastolic and mean arterial pressure, day-time mean arterial pressure and fall in nocturnal blood pressure $(-17 \%$ and $-18 \%$ respectively). Heart rate and all parameters of $L V$ mass were similar in patients and controls. The only alteration found in patients was in significantly greater carotid intimal-medial thickness (IMT) $(P<0.001)$. Atherosclerotic plaques were more frequent in patients than in controls, with a difference reaching a trend $\left(40 \%\right.$ vs $\left.10 \%, \chi^{2}=4.8 ; P=0.091\right)$. Considering that the carotid IMT is considered to be a marker of systemic atherosclerosis, our finding suggests early atherosclerotic changes in PHPT. No correlation was found between the severity and cardiovascular manifestation of PHPT.

Conclusions: Vascular changes may occur due to a combination of structural and functional impairments in PHPT patients, likely as a result of altered calcium metabolism and impaired equilibrium of other factors regulating vascular function. Both extent and duration of PHPT can play a relative role in the development of cardiovascular complications. Considering that PHPT is now recognized as a quite common and often symptomless endocrine disorder, the evidence of cardiovascular manifestation in normotensive patients, found by this morphological study, suggests a possible implication for the management of such patients. In this light, screening for abnormalities in cardiovascular system function should be recommended in all PHPT subjects.
\end{abstract}

European Journal of Endocrinology 147 453-459

\section{Introduction}

Despite the increasing evidence that primary hyperparathyroidism (PHPT) may contribute to greater risk of cardiovascular mortality and morbidity $(1-8)$, the etiopathogenetic mechanisms underlying this association are still not completely clear. Several abnormalities have been found in association with PHPT: arterial systolic hypertension $(1-6,9,10)$, premature atherosclerosis (11), valve calcifications (3, 5, 6), left ventricular (LV) hypertropy $(5,6)$ and triggered arrhythmia (3). However, the exact role of PHPT in the development of cardiovascular changes, its extent and clinical significance are still controversial (12). 
Several mechanisms seem to play a role in the association between PHPT and cardiovascular risk: high serum calcium levels (13), increased circulating parathyroid hormone (PTH) levels (14), enhanced activity of the renin-angiotensin system (15) and vascular wall function impairment $(16,17)$. While acute administration of PTH has a vasodilator effect (18), prolonged exposition to exogenous PTH led to increased blood pressure levels in healthy volunteers (19). This finding suggests that the variable 'time of exposition' can also play an important role in the influence on the cardiovascular system.

Clinical presentation of PHPT has changed in recent decades due to the large availability of routine serum calcium measurements, and PHPT is often discovered by chance. The clinical features of PHPT are mostly mild and sometimes totally asymptomatic (10, 12); however, an accurate evaluation can reveal some subclinical pathologies including cardiovascular system impairment, one of the most important target systems of PTH action $(20,21)$.

Although arterial hypertension is not considered a typical symptom of PHPT, its presence has been reported with a prevalence varying from $10 \%$ to $70 \%$ $(22,23)$. The increase in blood pressure (BP) is one of the most important factors involved in the development of LV hypertropy $(12,24)$ and average 24-h blood pressure levels and variability were found to correlate positively with severity of target organ damage (25, 26). On the other hand, LV hypertropy has also been documented in PHPT patients without hypertension and was reversed after successful parathyroidectomy (4). Finally, the lack of nocturnal fall (dipping) in BP has been observed to be strictly related to the increase in LV mass (27), that may contribute to cardiovascular damage (25).

Given the multiple influence of PHPT on the cardiovascular system, the present study aimed to assess the effects of PHPT on blood pressure profile, left ventricle mass and arterial vessels anatomy in patients with PHPT lacking typical symptoms and being normotensive.

\section{Subjects and methods}

\section{Subjects}

Forty consecutive patients, 16 men and 24 women, with a median age of 54 years (range 42 to 66 years) were evaluated at the Department of Molecular and Clinical Endocrinology and Oncology, of the 'Federico II' University in Naples for untreated PHPT and its complications.

Twenty patients were excluded for having hypertension (as detected according to the WHO guidelines, 5th Report of the Joint National Committee on High Blood Pressure 1993) $(n=13)$, increase in pancreatic enzymes $(n=1)$, impaired kidney function $(n=2)$, coronary artery disease $(n=2)$, or previous neck surgery $(n=2)$. The remaining 20 patients $(8$ males and 12 females), with a median age of 51.5 years (range 44 to 65 years) were included.

The diagnosis of PHPT was made on the basis of biochemical analysis: all patients had persistently increased serum calcium levels as well as inappropriately high levels of intact molecule PTH (Table 1). Moreover, reduced bone mineral density (osteopenia/ osteoporosis) (28) was found in eight patients, while micro-nephrolithiasis was detected by ultrasonography in nine. The mean time interval between the first documentation of hypercalcemia (invariably during follow-up of unrelated non-malignant disorders such as biliary colic, abdominal surgery or routine periodical check-up) and the study recruitment was 10 months (range 5-16 months). Fourteen of the participants fulfilled the indications for surgical treatment of PHPT (29). Twenty healthy individuals with normal blood pressure values, matched for age, gender and body mass index (BMI, $\mathrm{kg} / \mathrm{m}^{2}$ ) were studied as controls. They were consecutively recruited among patients referred to our department for thyroid ultrasonography who were not found to be affected by any relevant pathology. One PHPT patient and two control subjects were mild cigarette smokers $(<10$ cigarettes a day). None of the subjects recruited had previously been surgically treated for PHPT, nor did any take drugs for cardiovascular diseases. All subjects gave their informed consent to participate and the study was designed according to the II Declaration of Helsinki.

\section{Methods}

Biochemical evaluation Serum levels of PTH, calcium (Ca), phosphate (Ph), alkaline phosphatase (AP),

Table 1 Clinical and biochemical features of enrolled subjects.

\begin{tabular}{lcc}
\hline Variables & $\begin{array}{c}\text { Patients } \\
(n=20)\end{array}$ & $\begin{array}{c}\text { Controls } \\
(n=20)\end{array}$ \\
\hline Age (years) & $52.9 \pm 9.3$ & $54.6 \pm 8.6$ \\
Female/Male & $12 / 8$ & $12 / 8$ \\
BMI $\left(\mathrm{kg} / \mathrm{m}^{2}\right)$ & $24.2 \pm 1.2$ & $23.8 \pm 0.9$ \\
Initial supine systolic BP (mm Hg) & $135.7 \pm 4.6$ & $134.3 \pm 2.5$ \\
Initial supine diastolic BP (mm Hg) & $82.1 \pm 2.4$ & $82.1 \pm 1.5$ \\
Serum total calcium (mg/dl) & $12.2 \pm 0.87^{\star *}$ & $9.1 \pm 0.3$ \\
lonized calcium (mmol/l) & $1.86 \pm 0.7^{\star *}$ & $1.3 \pm 0.05$ \\
Urinary calcium excretion (mg/24h) & $357 \pm 120^{\star *}$ & $170 \pm 38$ \\
Serum phosphate $(\mathrm{mg} / \mathrm{dl})$ & $2.5 \pm 0.4^{\star *}$ & $3.4 \pm 0.6$ \\
Serum alkaline phosphatase (U/l) & $186.5 \pm 48^{*}$ & $140.4 \pm 36$ \\
Serum creatinine (mg/dl) & $0.8 \pm 0.09$ & $0.78 \pm 0.1$ \\
Intact molecule PTH (ng/l) & $280 \pm 165^{\star *}$ & $45 \pm 12$ \\
Known PHPT duration (months) & $11 \pm 4$ & - \\
& &
\end{tabular}

BMI, body mass index; PTH, parathyroid hormone; PHPT, primary hyperparathyroidism. Conversion factors to SI units: serum total calcium 0.2495. The known duration of the disease means the period between the first detection of hypercalcemia and the study entry.

${ }^{\star} P<0.05 ;{ }^{*} P<0.001$ vs controls. 
creatinine and 24-h urinary Ca excretion were determined. Total and high density lipoprotein (HDL) cholesterol, triglycerides, fasting glucose and insulin levels were within the normal range in all subjects and were similar in patients and controls.

Cardiovascular system evaluation Initially, a physical examination was performed and blood pressure was measured by the auscultatory method after a 20 -min rest in a supine position, according to the recommendations of the American Heart Association (30).

\section{Twenty-four-hour blood pressure profile} assessment Blood pressure monitoring was performed using an automatic recorder (ICR 90202, Spacelabs, Redmont, WA, USA). This system has previously been validated (26) and its accuracy was within the guidelines set by the British Hypertension Society (25). Patients were equipped with the device between 0800 and $0900 \mathrm{~h}$ on the non-dominant arm. Blood pressure and heart rate were detected every $15 \mathrm{~min}$ for $24 \mathrm{~h}$. Three systolic and diastolic BP values provided by the Spacelabs device were compared with those simultaneously obtained by standard sphygmomanometer, to ensure a difference within $5 \mathrm{~mm} \mathrm{Hg}$. Patients were recommended to perform their usual activities and to record them, including the time of waking up, going to bed and nocturnal awakening. Mean value of BP for the awake (day-time) and asleep (night-time) periods were thus defined. The average length of the night-time period was $8 \mathrm{~h}$. Neither loss of BP recordings nor the artifacts exceeded $10 \%$ of total measurement in any subject, and nobody reported significant night awakening. Mean arterial pressure values, determined as the mean of diastolic $\mathrm{BP}+1 / 3$ (systolic $\mathrm{BP}$-diastolic $\mathrm{BP}$ ), and nocturnal BP fall, determined as percent decrease in mean daytime pressure, were calculated.

Echocardiographic assessment Two-dimensional M-mode and Doppler echocardiography was performed with the standard techniques previously described (31) using Hewlett Packard model 77020AC with a $2.5 \mathrm{MHz}$ transducer (Hewlett Packard Co., Palo Alto, CA, USA). Images were obtained under sectorscanning monitoring to detect any change in $\mathrm{LV}$ shape and avoid angulation of the ultrasonic beam. Subjects were positioned in partial left decubitus. Simultaneous visualization throughout the cardiac cycle of the interventricular septal thickness (IVSTh), LV internal dimension (LVID), and posterior wall thickness (PWTh) was sought at or just below the tips of the mitral valve leaflets. Echocardiographically measured volumes in end-diastole and end-systole were derived using the cube formula (32) according to the simple anatomically validated formula $\mathrm{LVM}=$ $1.04 \times\left[(\text { IVSTh }+ \text { LVID }+ \text { PWTh })^{3}-\right.$ LVID $\left.^{3}\right]$ $-13.6 \mathrm{~g}$ (26), where LVM stands for left ventricle mass. To minimize the impact of variation in body size on LVM, correlation was made for body surface area and LV mass index (LVMI) was obtained. All measurements were performed according to the Penn Convention (32). The echocardiographic tracings were read and coded blind, in random order, always by the same two expert operators. Differences between readers of $<1 \mathrm{~mm}$ in the measurements of IVSTh and PWTh and of $<2 \mathrm{~mm}$ in the measurement of LVID were averaged; greater differences were resolved by reviewing the coded echocardiograms. The coefficient of variation for the different echocardiographic parameters was below 5\% (26).

\section{Ultrasound (US) carotid artery evaluation Imaging} was performed by echo-Doppler ultrasonography carried out with a $10 \mathrm{MHz}$ annular phased array transducer. Segments of right and left carotid arteries were scanned longitudinally (common, proximal internal carotid arteries and the carotid bulb). The US scanning was performed in the supine position after a rest of $10 \mathrm{~min}$. M-mode images were taken for several cardiac cycles, after achieving a satisfactory B-mode image of all segments. The site of greatest intimal-medial complex thickness (IMT) was scanned thoroughly from 3 angles: anterolateral, lateral and posterolateral. Each measurement was performed three times and the mean of all values obtained was calculated. The IMT was considered normal when $<1 \mathrm{~mm}$, indicative of arteriosclerosis when it ranged between $1 \mathrm{~mm}$ and $1.3 \mathrm{~mm}$ and indicative of stenosis when greater than $1.3 \mathrm{~mm}$ (33). The presence, size and localization of plaques were evaluated at the levels of all segments considered. The measurements were performed always by the same operator blinded to the patient or control status; the images were stored on magnetic media and analyzed later, when the data for all subjects were available.

Assays Blood chemistry profile, including levels of Ca, $\mathrm{Ph}$, AP, 24-h urinary calcium excretion, creatinine, cholesterol, triglycerides, and glucose were analyzed using a standard autoanalyzer. The reference range was $8.8-10.2 \mathrm{mg} / \mathrm{dl}$ for serum Ca, $2.5-4.8 \mathrm{mg} / \mathrm{dl}$ for serum $\mathrm{Ph}, 98-275 \mathrm{U} / \mathrm{l}$ for serum $\mathrm{AP}$ and $50-300 \mathrm{mg} / 24 \mathrm{~h}$ for urinary Ca excretion. Intact PTH was measured by radioimmunoassay (iPTH, Nichols Institute Diagnostic, San Juan Capistrano, CA, USA); reference range, $10-75 \mathrm{ng} / \mathrm{l}$. Conversion factors to SI units were: serum total calcium 0.2495; phosphorus 0.322 and creatinine 88.4.

Statistical analysis The values are expressed as means \pm S.D. The differences between the groups were evaluated by the paired $t$-test. Relationships were assessed by the Pearson's correlation coefficients and linear regression analysis. $\chi^{2}$ test was used to compare the frequency of the atherosclerotic plaques among 
patients and controls. A $P$ value was considered significant when $<0.05$.

\section{Results}

\section{Clinical and biochemical evaluation}

No abnormal finding was found at the physical examination. The clinical and biochemical data of the subjects enrolled are shown in Table 1. The group of patients had significantly higher $\mathrm{Ca}, \mathrm{AP}$ and $\mathrm{PTH}$ levels, higher $\mathrm{Ca}$ urinary excretion and lower $\mathrm{Ph}$ values. PTH levels ranged from 172 to $454 \mathrm{pg} / \mathrm{ml}$ and Ca from 11.4 to $13.5 \mathrm{mg} / \mathrm{dl}$ (Table 1 ).

\section{Twenty-four-hour blood pressure profile assessment}

No difference was found between the groups in terms of blood pressure profile when the following parameters were considered: supine systolic/diastolic pressure, average 24-h systolic, diastolic and mean arterial pressure. Day-time mean arterial pressure and fall in nocturnal blood pressure observed in the patients were also not significantly different as compared with healthy subjects $(-17 \%$ and $-18 \%$ respectively $)$ (Fig. 1).

\section{Echocardiographic assessment}

Heart rate and LV mass parameters, including LVM, IVSTh, PWTh and LVID, were similar in both patients and controls (Table 2). No significant calcification was found within the heart.

\section{Ultrasound carotid artery evaluation}

Patients' IMT ranged from $0.7 \mathrm{~mm}$ to $2.05 \mathrm{~mm}$. They had significantly greater carotid IMT $(P<0.01)$ and

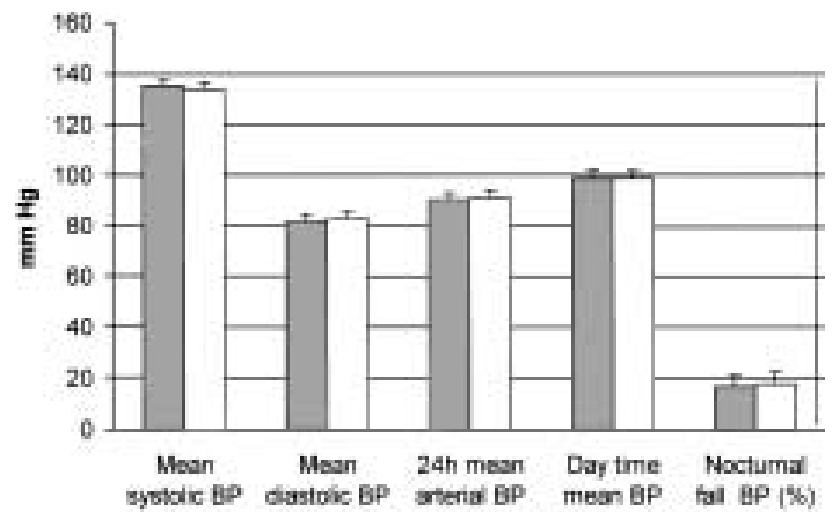

Figure 1 Blood pressure (BP) profile characteristics in patients (shaded bars; $n=20$ ) and controls (open bars; $n=20$ ). No significant difference was found between the two groups in any considered parameter. higher atherosclerotic plaques frequency than controls, the latter however did not reach statistical significance $\left(\chi^{2}=4.8 ; P=0.091\right)$ (Table 2$)$. When considering the patients individually, the IMT indicated atherosclerosis in $6(30 \%)$ patients and stenosis in $3(15 \%)$. The plaques were mostly situated at the carotid bifurcation, their thickness was $0.28 \pm 0.9 \mathrm{~cm}$ and their length was $2.2 \pm 1 \mathrm{~cm}$ (Fig. 2).

No significant correlation was found between mean 24-h BP, either diastolic or systolic, echocardiographic findings, mean IMT and the clinical features of the patients (age, estimated disease duration, values of PTH and serum $\mathrm{Ca}$, and Ca excretion).

Fourteen patients underwent surgery for PHPT. At surgical exploration, parathyroid adenomas were found in 13 (93\%) patients, and parathyroid hyperplasia resulted in one with a positive family history for PHPT, without other recurrent endocrine disorders.

\section{Discussion}

The increased frequency of early PHPT diagnosis by common screening tests has found a group of patients with mild or no typical symptoms of the disease (12); however, subtle cardiovascular, neurological and psychiatric impairments were described in many 'asymptomatic' patients $(21,34)$. As the clinical manifestations become subtler over time, the investigation has focused on less clinically overt abnormalities $(12,16,17)$.

This cross-sectional study evaluated the cardiovascular manifestation in a group of 20 normotensive patients affected by PHPT; no significant alteration in blood pressure profile and of LV mass was found, despite moderately high serum Ca levels. A satisfactory blood pressure profile with a physiological nocturnal fall was shown. However, the patients were shown to be mostly symptomatic for PHPT by appropriate evaluation (osteoporosis/osteopenia, kidney micro-lithiasis) and had increased carotid wall thickness.

Table 2 Ultrasonographic evaluation of the left ventricle mass and carotid wall intimal-medial thickness.

\begin{tabular}{lccr}
\hline Variable & $\begin{array}{c}\text { Patients } \\
(n=20)\end{array}$ & $\begin{array}{c}\text { Controls } \\
(n=20)\end{array}$ & $\boldsymbol{P}$ value \\
\hline LVID $\left(\mathrm{mm} / \mathrm{m}^{2}\right)$ & $48.4 \pm 2.36$ & $48.5 \pm 1.0$ & 0.868 \\
IVSTh $(\mathrm{mm})$ & $9.9 \pm 0.3$ & $10.1 \pm 0.5$ & 0.133 \\
PWTh $(\mathrm{mm})$ & $9.4 \pm 0.7$ & $9.4 \pm 0.3$ & 1 \\
LVMI $\left(\mathrm{g} / \mathrm{m}^{2}\right)$ & $113.4 \pm 13.3$ & $110.7 \pm 11$ & 0.488 \\
HR $(\mathrm{beats} / \mathrm{min})$ & $76 \pm 15$ & $74.5 \pm 14$ & 0.746 \\
IMT left $(\mathrm{mm})$ & $1.5 \pm 0.6$ & $0.7 \pm 0.3$ & $<0.001$ \\
IMT right $(\mathrm{mm})$ & $1.6 \pm 0.5$ & $0.68 \pm 0.3$ & $<0.001$ \\
Carotid artery plaques $(+/-)$ & $8(40 \%)$ & $2(10 \%)$ & 0.091
\end{tabular}

LVID, left ventricle internal dimension; IVSTh, interventricular septum thickness; PWTh, posterior wall thickness; LVMI, left ventricular mass index; HR, heart rate; IMT, intima-media thickness of the right and left common/ internal carotid artery wall. 



Figure $2 \mathrm{M}$-mode ultrasonography showing an example of a plaque detected at the right common carotid artery in one patient. A longitudinal scan (a) and a transverse scan (b) show a hypoechoic fibrolipid plaque along the anterior wall (arrowed); its thickness was $0.33 \mathrm{~cm}$ and the length was about $3 \mathrm{~cm}$.

Although hypertension and cardiovascular complications are not considered the most typical symptoms of PHPT, they were found in a significant percentage of patients $(1,3,8)$. Different hypotheses have been raised and several factors have been suggested to explain this association. Higher serum Ca levels were considered an independent predictor of mortality in humans (12). A direct relationship between PTH, LV hypertropy and vascular resistance has also been shown (35). Furthermore, differences have been found in the clinical manifestations of mild and severe PHPT $(20,36,37)$, although some data are still controversial. Stefenelli et al. (8) have reported LV hypertropy, and valve and myocardial calcifications in a significant number of patients with mean serum calcium levels of $12 \mathrm{mg} / \mathrm{dl}$. In contrast, patients who had a milder increase in serum $\mathrm{Ca}(11.1-11.3 \mathrm{mg} / \mathrm{dl})$ were found to have only LV hypertropy but no significant valvular or myocardial calcification $(38,39)$. On the other hand, Längle et al. did not find any direct correlation between the severity and the cardiovascular manifestations of PHPT; however, PTH-related skeletal manifestations and valvular calcifications were predictive factors for left ventricular hypertropy (37). In a study by Lindt et al. (36), mild PHPT was also associated with an increase in BP, which persisted both in subjects successfully treated by surgery and in those who did not undergo surgery. The inefficacy of surgery on hypertension in PHPT may indicate that the development of hypertension is neither related directly to hypercalcemia nor to the high PTH levels, but to yet unknown factors or to a combination of factors. Another hypothesis is that PHPT was diagnosed and treated too late in hypertensive patients. Most patients may have had PHPT for years before being diagnosed as such; the persisting disease might be responsible for triggering a multifactorial irreversible process leading to manifest hypertension (40). Mean calcium levels were $12.2 \mathrm{mg} / \mathrm{dl}$ in our patients and the estimated duration of hypercalcemia was 10 months. It is intriguing that many parameters of the cardiovascular system evaluated by this study were normal, despite high levels of calcemia, similar to those of the Stefenelli et al. study (8). Differences in the cardiovascular manifestation could likely be related to different duration of disease. The lack of correlation in the present study between patients' BP values on the one hand and their clinical and biochemical features on the other is in line with these previous data, suggesting no direct relationship between BP behavior and disease activity (expressed as circulating levels of calcium and PTH).

The only detectable alteration of the cardiovascular system in this study was a significant increase in IMT of the carotid arteries, compared with controls. Since carotid IMT is recognized as a marker of systemic atherosclerosis (38), our patients can be considered as having premature atherosclerosis, which was also confirmed by higher prevalence of atherosclerotic plaques compared with controls ( $40 \%$ vs $10 \%$ ), although the difference did not reach significance due to the relatively small population under evaluation. Increased IMT indicates reduced diameter and significantly increased stiffness of carotid walls, the latter being recognized as an independent marker of cardiovascular risk $(41,42)$. This result is in accordance with Smith et al., who have found significantly higher indices of vessel stiffness in PHPT patients compared with healthy controls, independently of systolic BP values (20). However, these authors used pulse wave analysis, which generates central arterial waveforms dependent on a 
number of factors, including age, height and obesity (12) and which may have influenced the results of the measurements. Moreover, those patients presented with other risk factors including significantly higher cholesterol, triglycerides and fasting insulin levels (12); therefore, it is not clear to what extent each factor contributed to the increased cardiovascular risk. In the present study, the premature atherosclerosis seems to be found independently of cholesterol, triglycerides and insulin values, suggesting a possible direct relationship between cardiovascular risk and PHPT. Increased vessel stiffness may be an initial change in PHPT patients, due to a combination of structural and functional vascular changes occurring as a result of altered calcium metabolism and PTH direct action on vascular smooth muscle and endothelial cells $(35,43)$.

Moreover, since acute PTH administration was described as having a different effect than the chronic one $(18,19)$, the clinical effects of PHPT on the cardiovascular system can differ according to disease duration. This is in line with previous results on cardiac abnormalities in a large population of PHPT patients (37). Both extent and duration of PHPT history may play a relative role in clinical manifestation of the disease.

In conclusion, the evidence of symptomless vascular abnormalities together with previous data on subclinical alteration of cardiovascular function suggests that all PHPT subjects should be evaluated for cardiovascular risk factors (44). Aggressive management of cardiovascular risk factors should be considered in all subjects regardless of the clinical manifestation.

\section{Acknowledgements}

We are indebted to Mrs Rosanna Scala for her help in editing the manuscript.

\section{References}

1 Heath H, Hodgson SF \& Kennedy MA. Primary hyperparathyroidism: incidence, morbidity and potential economic impact in a community. New England Journal of Medicine $1980 \mathbf{3 0 2}$ 189-193.

2 Hedback G \& Oden A. The increased risk of death from primary hyperparathyroidism - an update. European Journal of Clinical Investigation $1998 \mathbf{2 8} 271-276$.

3 Rayner HC \& Hoskins DJ. Hyperparathyroidism associated with severe hypercalcemia and myocardial calcification despite minimal bone disease. British Medical Journal $1986 \quad 293$ 1277-1278.

4 Stefenelli T, Abela C, Frank H, Koller-Strametz J \& Niederle B. Time course regression of left ventricular hypertrophy after successful parathyroidectomy. Surgery $1997 \mathbf{1 2 1} 157-161$.

5 Niederle B, Stefanelli T, Glogar D, Wloszczuk W, Roka R \& Mayer H. Cardiac calcific deposits in patients with primary hyperparathyroidism: preliminary results of an echocardiographic study. Surgery $1990 \mathbf{1 0 8} 1052-1057$.
6 Katz JH, Dias SM \& Ferguson RP. Fatal cardiac calcifications secondary to primary hyperparathyroidism. American Journal of Medicine $1988 \mathbf{8 5} 122-123$.

7 Bikkina M, Levy D, Evans J, Larson MG, Benjamin EJ, Wolf PA et al. Left ventricular mass and risk of stroke in an elderly cohort. The Framingham heart study. JAMA 1994272 33-36.

8 Stefenelli T, Mayr H, Bergler-Klein J, Globits S, Woloszczuk W \& Niederle B. Primary hyperparathyroidism: incidence of cardiac abnormalities and partial reversibility after successful parathyroidectomy. American Journal of Medicine 199395 197-202.

9 Levy D, Garrison R, Savage DD, Kannel WB \& Castelli WP. Prognostic implications of echocardiographically determined left ventricular mass in the Framingham heart study. New England Journal of Medicine 1990332 1561-1566.

10 Lafferthy FW. Primary hyperthyroidism. Changing clinical spectrum, prevalence of hypertension, and discriminant analysis of laboratory tests. Archives of Internal Medicine $1981 \mathbf{1 4 1}$ $1761-1766$.

11 Roberts WA \& Waller BF. Effect of chronic hypercalcemia on the heart: an analysis of 18 necroscopy patients. American Journal of Medicine 198171 371-384.

12 Silverberg SJ. Cardiovascular disease in primary hyperparathyroidism. Journal of Clinical Endocrinology and Metabolism 2000 $853513-3514$.

13 Leifsson BG \& Ahren B. Serum calcium and survival in a large health screening program. Journal of Clinical Endocrinology and Metabolism $2000 \mathbf{8 1} 2149-2153$.

14 Ogino K, Burkoff D \& Bilezikian JP. The hemodynamic basis for the cardiac effect of parathyroid hormone and parathyroid-hormone related protein. Endocrinology $19951363024-3030$.

15 Horky K, Broulik P \& Pacovsky V. The effect of parathyroid hormone on plasma renin activity in humans and hypertension in patients with primary hyperparathyroidism. Journal of Hypertension 19864 S585-S587.

16 Neunteufl T, Katzenschlager R, Abela C, Kostner K, Niederle B, Weidinger $\mathrm{F}$ et al. Impairment of endothelium-independent vasodilatation in patients with hypercalcemia. Cardiovascular Research 199840 396-401.

17 Nilsson IL, Aberg J, Rastad J \& Lind L. Endothelial vasodilatory dysfunction in primary hyperparathyroidism is reversed after parathyroidectomy. Surgery $1999 \mathbf{1 2 6} 1049-1055$.

18 Bukoski RD, Ishibashi K \& Bian K. Vascular action of calcium regulating hormones. Seminars in Nephrology $1995 \quad 15$ 536-549.

19 Hulter HN, Melby JC, Peterson JC \& Cooke CR. Chronic continuous PTH infusion results in hypertension in normal subjects. Journal of Clinical Hypertension 19864 360-370.

20 Smith JC, Page MD, John R, Wheeler MH, Cockcroft JR, Scanlon MF et al. Augmentation of central arterial pressure in mild primary hyperparathyroidism. Journal of Clinical Endocrinology and Metabolism 200085 3515-3519.

21 Barletta G, De Feo ML, Del Bene R, Lazzeri Ch, Vecchiarino S, La Villa G et al. Cardiovascular effects of parathyroid hormone: a study in healthy subjects and normotensive patients with mild primary hyperparathyroidism. Journal of Clinical Endocrinology and Metabolism $2000 \mathbf{8 5} 1815-1821$.

22 Rapado A. Arterial hypertension and primary hyperparathyroidism. Incidence and follow-up after parathyroidectomy. American Journal of Nephrology 19866 (Suppl 1) 49-50.

23 Silverberg SJ, Shane E, Jacobs TP, Siris E \& Bilezikian JP. The natural history of treated and untreated asymptomatic primary hyperparathyroidism. New England Journal of Medicine 1999 341 1249-1255.

24 Stefenelli T, Abela C, Frank H, Koller-Strametz J, Globits S, BerglerKlein J et al. Cardiac abnormalities in patients with PHPT: implications for follow-up. Journal of Clinical Endocrinology and Metabolism 199782 106-112.

25 Dimsdale JE, von Kanel R, Profant J, Nelson R, Ancoli-Israel S \& Ziegler M. Reliability of nocturnal blood pressure dipping. Blood Pressure Monitoring 20005 217-221. 
26 Fontana D, Izzo R, Trotta R, Iovino GL, Argenziano L, Fratta L et al. Blood pressure profile as a predictor of reversal of cardiovascular structural changes during antihypertensive treatment. Current Therapeutic Research 199758 108-115.

27 Verdecchia P, Schillaci G, Guerrieri M, Matteschi C, Benemio G, Boldrini F et al. Circadian blood pressure changes and left ventricular hyperthrophy in essential hypertension. Circulation $199081528-536$.

28 World Health Organization, Assessment of fracture risk and its application to screening for postmenopausal osteoporosis. WHO Technical Report Series 843. Geneva: WHO, 1994.

29 NIH Conference: diagnosis and management of asymptomatic primary hyperparathyroidism: consensus development conference statement. Annals of Internal Medicine 199114 593-597.

30 Kirkendall WH, Burton HC, Epstein FH \& Freis ED. Recommendation for human blood pressure determination by sphygmomanometers. Circulation 196736 980-988.

31 Trimarco B, Ricciardelli B, De Luca N, De Simone A, Cuocolo A, Galva MD et al. Participation of endogenous catecholamines in the regulation of the left ventricular mass in progeny of hypertensive patients. Circulation 198572 38-46.

32 Deveraux RB \& Reichek N. Echographic determination of left ventricular mass in man. Anatomic validation of the method. Circulation 197755 613-618.

33 Kawasaki T, Sasayama S, Yagi SI, Asakawa T \& Tadakazu H. Non-invasive assessment of age-related changes in stiffness of major branches of the human arteries. Cardiovascular Research $198721678-687$.

34 Clark OH, Wilkes W, Siperstein AE \& Duh Q-Y. Diagnosis and management of asymptomatic hyperparathyroidism: safety, efficacy, and deficiencies in our knowledge. Journal of Bone and Mineral Research 19916 S135-S142.

35 Nickols GA. Action of parathyroid hormone in the cardiovascular system. Blood Vessels 198724 120-124.

36 Lindt L, Jacobsson S, Palmér M, Lithell H, Wengle B \& Ljunghall S. Cardiovascular risk factors in primary hyperparathyroidism: a 15-year follow-up of operated and unoperated cases. Journal of Internal Medicine $199123029-35$.

37 Längle F, Abela C, Koller-Strametz J, Mittelbock M, Bergler-Klein J et al. Primary hyperparathyroidism and the heart: cardiac abnormalities correlated to clinical and biochemical data. World Journal of Surgery $1994 \mathbf{1 8} 619-624$.

38 Dahlberg K, Brodin LA, Juhlin-Dannfelt A \& Farnebo LO. Cardiac function in primary hyperparathyroidism before and after surgery. European Journal of Surgery $1996 \mathbf{1 6 2} 171-176$.

39 Piovesan A, Molineri N, Casasso F, Emolo I, Ugliengo G, Cesario F et al. Left ventricular hypertrophy in primary hyperparathyroidism. Effects of successful parathyroidectomy. Clinical Endocrinology $199950321-328$

40 Jorde R \& Sundsfjord J. Bone mineral density and blood pressure in patients with asymptomatic hyperparathyroidism. The Thromsø Study. Journal of Internal Medicine $2000 \quad \mathbf{2 4 7}$ 325-330.

41 Arnett DK, Evans GV \& Riley WA. Arterial stiffness: a new cardiovascular risk factor. American Journal of Epidemiology $1994140669-682$.

42 Glasser SP, Arnett DK, McVeigh GE, Finkelstein SM, Bank AJ, Morgan DJ et al. Vascular compliance and cardiovascular disease: a risk factor or a marker. American Journal of Hypertension 1997 10 1175-1189.

43 Hanson AS \& Linas SL. Parathyroid hormone/adenylate cyclase coupling in vascular smooth muscle cells. Hypertension 1994 $23468-475$.

44 Nuzzo V, Fonderico F, Caserta A, Fittipaldi MR, Fontana D \& Barbato E et al. Evaluation of 24-h blood pressure behavior in normotensive hyperparathyroidism. In Congress on Hormones and Heart, Naples, 24-26 September 1998. Abstract 75.

Received 10 January 2002

Accepted 5 July 2002 